\title{
CASES AND OBSERVATIONS
}

CONNECTED WITH

DISE ASE

OF THE

\section{PANCREAS AND DUODENUM,}

\author{
BY RICHARD BRIGHT, M.D. F.R.S. \\ FELLOW OF THE ROYAL COLLEGE OF PHYSICIANS, LECTURER ON \\ THE PRACTICE OF MEDICINE, AND ONE OF THE PHYSICIANS \\ TO GUY'S HOSPITAL. \\ READ NOVEMBER 1 STH, 1832.
}

Altrougr the cases and observations which I am about to lay before the Society, are stated to be connected with derangement of the pancreas and duodenum; yet they are chiefly intended to call the attention of the Members to a particular symptom in disease, which I believe to have been but little noticed. In this I may be mistaken, but at all events it is not a frequent symptom, and the present paper will serve as an additional record. of its existence, even though I should fail, as I fear I shall, to bring forward any thing conclusive in elucidation of the morbid state, or even demonstrative of the organ, on which it depends, and I am the less scrupulous in submitting these cases to the Society because they are not without interest, independently of the direct VOL. XVIII. 
reference to the subject for which they are introduced.

The symptom to which I refer is a peculiar condition of the alvine evacuation, a portion more or less considerable assuming the character of an oily substance resembling fat, which either passes separately from the bowels, or soon divides itself from the general mass, and lies upon the surface, sometimes forming a thick crust, particularly about the edges of the vessel, if the fæces are of a semi-fluid consistence, sometimes floating like globules of tallow which have been melted and become cold, and sometimes assuming the form of a thin fatty pellicle over the whole, or over the fluid parts, in which the more solid figured fæces are deposited.

This oily matter has generally a slight yellow tinge, and a most disgustingly fetid odour. I submitted a portion of it to Dr. Bostock for examination, so long ago as the year 1828; and he declared it to be adipocire. I shall, however, in the observations I make, refer less to the chemical properties of the substance than to the obvious symptom, - the existence of the separated matter resembling fat, which is still no less a marked symptom whether it be found to be a new secretion; or only a modification of that fatty matter of which Berzelius has spoken as being one of the component parts of fæces, and is in the natural and healthy state completely mingled with 
them; or whether it be some new compound produced from the alimentary matter.

The first case which I bring forward because the first in which I had an opportunity of following out this peculiar symptom by an examination after death, I shall relate in the words of my friend Dr. Benjamin Babington, with whom I saw the patient several times during life, and assisted at the post mortem examination.

\section{$C A S E I$.}

Diabetes,-with supervening icterus from obliteration of the common bile duct, caused by disease of the pancreas,-malignant ulceration of the duodenum,-copious discharge of fatty matter with the dejections.

James Barnes, aged 49, clerk in a waggon office, a man of sober and regular habits, began to complain, in March 1827, of immoderate thirst and appetite, with a constant pain in his loins. He made water very frequently, and his urine was of a pale colour. These symptoms accompanied by emaciation increasing upon him, he applied for advice successively to several medical men. It was not, however, until the middle of August following, that his disorder was pronounced to be diabetes mellitus. In the beginning of September he first became affected with jaundice, but his change of colour was not pre- 
ceded by any painful sensations. Under different Dispensaries and practitioners, he was subjected to various medical treatment, until the 4th of December, the period at which I first saw him. He was then reduced to a state of great debility, although still by no means a thin man. His thirst and appetite were constant, his pulse was 80 and soft, his skin deeply tinged with bile. His urine, of which he made about nine pints in $\mathbf{2 4}$ hours, was deep coloured, and left a yellow stain on linen. It was very sweet, and had a specific gravity of 1039 . His evacuations by the bowels were copious and light coloured. The liver was at this time but obscurely felt through the integuments, as these were still filled out with adipose matter. My directions were that the patient should live upon animal food with a moderate portion of greens or lettuces, and that he should eat as little bread as possible, and avoid all kinds of roots, fruits, and such articles of diet as contain sugar or meal; that he should drink tea without sugar, and at his dinner stale porter or port wine and water. With his diet thus regulated, a bitter aromatic mixture with subcarbonate of soda was prescribed.

On the 18th of December he began to keep an account of the quantity of liquid drunk and of urine made in the 24 hours, prefacing it with a detail of the nourishment taken on that day, and this he continued, with the exception of one week, until he grew too much debilitated to write. 
Between the 5th and the 18th of December, the urine gradually diminished in quantity and specific gravity, and on the latter date the diabetic character of the complaint was so far subdued, that attention was now more particularly directed to the obstruction of the bile. With this view, the following pill was directed, as an addition to the mixture already prescribed.-

R Pilul. Hydrag. Эi.

Extr. Taraxaci Эij.

Contunde et divide in Pilulas duodecim quarum sumat unam ter quotidie.

From this period I saw Mr. Barnes at intervals, and made the following remarks on these occasions. On the 24th of December the skin continued tinged of a bright yellow colour, the bowels were regular. The urine was somewhat less highly coloured than on the preceding week. It had entirely lost its sweet taste and its specific gravity was only 1015, or rather under than beyond the healthy standard. The quantity of liquid taken on this day was six pints, and the quantity of urine made amounted to only four. From this time forth the patient might be said to have lost altogether the diabetic disorder, or, at least that sign of it which consists in the formation of sugar in the urine. On a more minute examination of the liver than I had before made, it was very distinctly felt, hard and solid, projecting three or four inches beneath the ribs. The edge could be made out 
to be rounded, the notch was perceptible to the touch, and though the parietes were not at this time very thin, the gall-bladder, enormously distended, could very plainly be felt through them. The mouth had been very sensibly affected by the mercurial for several days past. It was now therefore discontinued and the taraxacum alone employed with the alkaline tonic mixture.

On Friday the 28th, without any notable change of symptoms, the patient began to pass a quantity of yellowish fatty matter much resembling butter that had been melted and had become again solid. This matter followed the fæces, and as it was evacuated in a melting state it was perceived on the surface of the dejection. We could not trace the origin of this change to any thing that had been eaten, for he had been upon a rigid system of diet from the time that he had been under my care, and had taken nothing of a fatty nature since that period. Meat, indeed, he had used in abundance, but his wife had constantly and carefully removed the fatty parts. For the subcarbonate of soda in the tonic mixture, liquor potassæ, in the proportion of 15 minims to the dose, was substituted to meet more effectually this symptom.

On December the 31st, I found that this alteration in the mixture had done away entirely the oily character of the motions, three of which, copious in quantity, were then passed in the 24 hours. The specific gravity of the urine was still 1015, but notwithstanding this amelioration in one part of his complaint, the 
patient was evidently growing weaker and more emaciated from the obstinate obstruction of the gall bladder, and the consequently defective assimilation of the nourishment. The taraxacum seemed, indeed, to supply the place of bile, so far as its merely stimulating effects and the consequent action of the bowels were concerned; but the evacuations which passed were of an unhealthy character, made up of half digested food, pasty, deficient in colour, and feculent odour, and more copious than they would have been, had the usual proportion of nutritive parts been abstracted. The effect of the mercury had nearly subsided, but pain being now felt in the hepatic region, a large blister was applied over that part, and the same medicine was continued.

On the 8th of January 1828, when I next saw my patient, he was somewhat more debilitated than on the preceding week, and still remained thoroughly jaundiced. He passed four or five stools in the 24 hours, somewhat relaxed, and now again containing some of the fatty matter already described, as well as portions of undigested meat. The appetite was good but not voracious. Sleep disturbed, seldom lasting more than an hour and a half at a time. Tongue somewhat brownish in the centre but moist, and, excepting this slight alteration in colour, not much deviating from the healthy state. Pulse 84, soft. Urine high coloured, specific gravity 1020. More bread had within the last day or two been added to the diet, which otherwise remained the 
same. With reference to the relaxed state of bowels and frequent motions, a chalk mixture with aromatic confection and tincture of opium was directed.

At the same time, in order that the liver might not be neglected, however hopeless the attempt to relieve its gorged state, a mercurial plaister was applied over the part, the painful sensations in which had been somewhat relieved by the blister.

On the 13th, I found that the number of dejections had been reduced to three in the 24 hours. Only two copious evacuations had been past on the preceding day, which continued of a pasty, stringy consistence, but were not of a very light colour. The patient was evidently more emaciated and duller than he had yet appeared, and the colour of his skin had not improved. The edge of the liver and large rounded extremity of the gall bladder were still plainly felt. The rest of the abdomen was flaccid. Severe pain was felt on the right passing through to the left side, which almost wholly prevented sleep. The tongue was clean and of a healthy colour, pulse 87, weak. Ordered to be cupped on the right side to eight ounces and to take four grains of blue pill with two grains of extract of poppies every night. On the following day the pain in the side was relieved. The quantity of urine made had been four pints in the $\mathbf{2 4}$ hours, while $5 \frac{1}{2}$ pints had been drunk, the specific gravity was 1020 , that of the bloody serum collected from the cupping 1030. On the 16th the bowels 
became again very much relaxed, five evacuations having been passed during the preceding night. The motions were of a dark colour and still contained a little oily matter, though less than had been observed the preceding fortnight. The appetite was still moderate and even precarious, the sleep much disturbed. The pills were directed to be discontinued, and a powerful astringent mixture, containing hæmatoxylum, catechu and opium, was ordered.

21st. Much weaker, yet still able to move slowly about the house. The bowels continued relaxed to the extent of six motions a day, and what passed was dark and undigested. The feet were now, for the first time, observed to be somewhat odematous, and the skin remained of a deep yellow hue. The emaciation was progressive, the resst continued much disturbed. There was now no complaint of thirst, nor indeed had this been the case since the change in the specific gravity and quantity of the urine; but the appetite was again increased.

From the beginning of February Mr. Barnes was evidently a dying man. On the 10th of that month he began to labour under symptoms of pleuritis in the left side. On the 26th, the quantity of urine made amounted to only $2 \frac{1}{2}$ pints. The appetite had now failed. The bowels, which had been hitherto relaxed, were confined, so that no motion had been obtained from the Sunday to the Tuesday, the specific gravity of the urine was only 1006, and, though what food was taken consisted 
entirely of farinaceous articles, it was not increased either in specific gravity or in quantity from this circumstance. He died without pain or struggle, sitting up in his bed, on the 1st of March, worn out with emaciation, debility, and want of rest, but retaining his senses, and a perfectly collected state of mind till the last moment of his life.

Sectio Cadaveris.-The limbs were remarkably flaccid, the whole skin was of a dark yellow colour; there was great general emaciation, and the legs were slightly œdematous.

Both the pleura costalis and pulmonalis were tinged with bile. The lungs were healthy in structure throughout, except the lower lobe on the left side, where very acute pleuritis had recently occurred, throwing out a thick coating of gelatinous fibrin, which broke down readily when the lungs were drawn forwards. This was partly deposited on the lower surface against the diaphragm, and partly on the posterior part of the ribs near their angle, and it was highly tinged with bile. The lung itself was implicated, and a small abscess of the size of an olive was formed near the edge, where it rests upon the diaphragm. The pericardium was deeply stained with bile, and the large vessels near the heart were quite yellow, this colour pervading their whole thickness. The heart was rather small and contracted, but retained its natural proportions, and the valves were healthy. The abdomen contained rather more 
than a gallon of dark olive coloured fluid. The gall bladder distended with very dark bile, was seen with its fundus projecting when the parietes were first removed. The liver was of a dark olive colour from the bile with which it was pervaded. The ducts were greatly enlarged. The common duct was large enough to admit the little finger freely when passed from above downwards, and its internal surface presented a honey-comb or reticulated appearance, and terminated by a cul de sac in the diseased substance of the pancreas, and at its shut end, a rough white deposit had taken place probably either of fibrin or cholesterine.

The head of the pancreas formed with some of the surrounding glands, a hard globular mass, round which the duodenum turned, and to which both it and the pylorus were firmly joined, and in two parts, where the pancreas and duodenum were welded together by the disease, ulcers of a hard and scirrhous character had taken place, penetrating the whole thickness of the intestine ; one of them of the size of a shilling, and the other not larger than a silver penny piece. The pancreas was hard and cartilaginous to the touch, and of a bright yellow colour.

A section of the liver looked like a fine grained dark greenstone porphyry, or very dark Aberdeen granite ; the ducts, which were throughout enlarged, being completely filled with bile which flowed from the incision. In different parts of the liver a few irre- 
gular masses occurred of a firm hard consistence, but shaded off into the substance of the liver, and not bearing the appearance of circumscribed tubera. The stomach was slightly vascular. The spleen natural in structure, but its external surface mottled, with cartilaginous deposit. The intestines were tolerably natural, but somewhat opake, and the internal lining rather pale. The kidneys were to external appearance perfectly healthy; but the tubular parts shewed themselves more plainly than usual, when the kidney was torn open, and in some of the tubes were white specks from a deposit either of fibrin or of calculous matter. The pelvis of the kidney was not vascular, but was tinged with bile. The lining membrane of the bladder was remarkably healthy and free from all vascularity; but its net-like appearance bespoke more than usual action in the muscular coat. The aorta and common iliacs were in many patches diseased with bony deposits surrounded by dark spots, where the internal surface had been destroyed by ulceration or absorption.

\section{CASE II.}

Icterus from obliteration of the common bile duct, caused by disease of the pancreas, malignant ulceration of the duodenum. Fatty matter in the dejections.

Elizabeth Tubbs, aged 50, living at Gravesend, was admitted into Guy's hospital under my care, November 19th, 1828. She had been for some time 
under the treatment of Mr. Beaumont, but her husband becoming anxious, determined to bring her to the hospital. She was at that time intensely yellow with jaundice, but the colour was rather of a dingy than of a brilliant hue. She was greatly emaciated, the skin of the face appearing to be drawn tight, and the cheeks sunk. She stated that about 17 years before, she was supposed to have had a severe inflammation of the liver, since which she had enjoyed good health; but had been subject to occasional retching and vomiting, even in her best health, and this had been more frequent and severe of late, often occurring half an hour after taking food, and sometimes when she stooped, as to tie her shoe. For the last four months her stools had been occasionally of a clay colour. Her skin had from time to time become yellow. Three months ago she was attacked with most violent pain in the lower part of her abdomen, attended with diarrhœa; her food passing in an undigested state. The severe pains still continued, at the time of her admission, to return at intervals, and were relieved by pressure, or by lying on her stomach. Pressure at the scrobiculus cordis, or on other parts of the abdomen gave no pain. The stools were very nearly white, and urine highly tinged with bile, staining her linen very much. Her skin had not been constantly of a yellow colour till the last month or six weeks. I immediately concluded from the gradual progress of the disease, from the deep dingy colour of the jaundice, the long continued occasional sickness, and the sunk countenance, that the disease 
was connected with some organic obstruction pressing at once on the gall ducts and the pylorus, which I entertained no hope of relieving. I determined however to give a little blue pill for a few days, with the carbonate and sulphate of magnesia, which latter I changed on the following day for the compound decoction of aloes, and the bowels being still rather costive, I ordered half an ounce of castor oil upon the 24th. On the following day I observed the stools to present one of those appearances which they sometimes derive from castor oil, being covered with white round masses of the size of peas and larger, and this I pointed out as being a change which the castor oil frequently undergoes in the intestines: but the woman hearing my observation, immediately said that she frequently passed an oily or greasy matter with her stools when she had taken no castor oil or other greasy substance, and she shewed me some of this now about the sides of the vessel, which looked like melted grease, exactly resembling what occurred in the case of Barnes, (Case I.,) but in smaller quantity.

December 1st. The symptoms were not materially altered, but the catamenia, which had hitherto been regular, appeared to-day, a fortnight after the usual period; and as I every day became more and more confirmed in my opinion, that the biliary obstruction depended on organic change, I determined to discontinue even the very limited quantity of blue pill which she had been taking; and she was ordered to have merely the nitric acid mixture for drink. 
5th. The catamenia nearly subsided. The discharge had its usual appearance, in no way altered by her complaint, as far as she could perceive. She had rather more pain than usual in her right side.

Habeat extracti taraxaci $\ni_{j}$ ter quotidie et repetatur mixtura acidi nitrici.

6th. I had an opportunity of again seeing some of the fatty matter in her dejections, and gave a portion to Dr. Bostock. As I had strictly forbidden her having either animal food or castor oil, in order that every source of fallacy might, as far as possible, be removed, she had taken neither for the last ten days, except occasionally a small quantity of broth and a little butter with her bread. These were the only sources from which the fatty matter could have been derived as far as diet was concerned, and she herself altogether rejected the idea of its depending on any thing taken in, as she said it was an occurrence she had very frequently observed since her present illness. The fatty matter formed a thick pellicle over some fluid surrounding a semi-fluid dejection, and formed hard lumps as thick as a penny-piece around the edges of the freces.

December 7th. She suffered considerable pain in the lower part of the abdomen, and had been sick at the stomach, vomiting a ropy grumous fluid.

Habeat infusi Gentian comp. Ziss c Sodæ subcarbo- 
natis gr. Xv. et extracti taraxaci Эj forma pilul. ter die.

I had this day, for the first time, an opportunity of conversing with the poor woman's husband, to whom I stated my conviction that the disease was of a fixed organic character and not capable of cure, although by carefully watching symptoms, much might be done to alleviate suffering. He therefore resolved to take his wife back to Gravesend, promising to let me know when the fatal termination should take place, as I was exceedingly anxious to see whether, in this case, the head of the pancreas itself and the duodenum were the seat of disease, as in the case of Barnes, where the same peculiar fatty matter had been passed from the bowels.

No very marked change took place after her return home, but she suffered more pain in the stomach and bowels, which was relieved by opiates. After a few weeks she passed some dark motions like pitch, and about a week before her death a considerable quantity of clear blood which formed large coagula. During the several last days of her life she became exceelingly drowsy, and for 24, hours before death slept almost constantly.

She died on the 16th of February, and as herhusband sent ue information immediately, I lost no time in repairing to Gravesend, where, with the kind assistance of Mr. Beaumont and Mr. Russell, a careful inspection 
took place. Judging from the history and progress of symptoms, and assuming the possibility that the peculiar fatty discharge might be connected with the same condition of the viscera, and probably of the pancreas and duodenum, as in the case of Barnes, I predicted that the appearances would correspond in the two cases, and certainly the result shewed a very remarkable similarity.

Sectio Cadaveris, Feb. 18, 1829. - Skin generally of a deep yellow colour, varying in parts to greenish brown, not very unlike the dark colour of the Creole. General emaciation, but by no means to the extent sometimes seen ; indeed on the abdomen, there was a considerable portion of fat of a deep yellow colour.

The lungs were in a very healthy state, except that they were both bound firmly at the posterior part by very strong adhesive bands, and the whole surface was tinged moderately with bile. Heart healthy, but small and not firm.

On opening the abdomen, the omentum rather loaded with fat. No peritoneal disease or adhesion, except on the superior surface of the liver which was attached in several parts by long adhesions to the diaphragm.

The cause of pressure on the bile ducts was immediately obvious; for on placing the hand near the pylovoL. XvIII. 
rus a hard lump of the size of a common egg was easily felt, and was soon discovered to be the head of the pancreas itself, and not the glands surrounding that part, forming a yellow mass like the boiled udder of a cow, almost cartilaginous. Its texture was uniformly hard and unyielding, and the whole pancreas partook of the same, but in a less degree. The head of the pancreas was firmly and inseparably glued to the duodenum, and the hardness very nearly surrounded that viscus. Laying open the duodenum, its internal surface was uneven and ulcerated, the ulcer having eroded the whole of the coats, and in the portion lying on the head of the pancreas it was of a soft consistence and light yellow colour, communicating with the substance of the tumour here irregularly softened or suppurating to the extent of a small chesnut. In the midst of the ulcer a little nipplelike body was seen projecting on its surface, which proved to be the orifice of the common duct of the gall bladder. This was still pervious as the thick bile could be squeezed out of the gall bladder through it. But it was obvious that this had either lately become pervious by the ulceration of its orifice, or the ulceration of the hard mass in which it was imbedded, or that its situation in the contracted duodenum had acted as a compressing cause; for the gall bladder was distended, containing at least four ounces of thick dark green bile which stained the lining membrane of the deepest colour. The gall bladder, though thus loaded, was not tonse, and conveyed the idea of a somewhat flaccid bag, so that I should have pro- 
nounced it to have been more distended lately than at the present moment. The disease of the mucous coat of the intestines occupied the outside of the ridge forming the pylorus, which was strongly marked, but was not scirrhous; yet on passing the finger before the pylorus was cut open, the hardened neighbouring structure produced all the effect of a stricture.

The liver was of its natural size, containing several round tubera sprinkled through various parts, from the size of a grain of rice to that of a nutmeg. These were not very numerous, but five or six were seen on the superior surface where they were perfectly circular, and a little depressed in the centre. They were decidedly harder than the surrounding liver, but did not separate freely from it; on the contrary, generally seemed to be shaded off into the surrounding parts and the texture of the liver was discernible in them. The larger tubera were soft and yellow in the centre. The general structure of the liver was healthy, rather soft, and of a dark olive green colour. The biliary ducts were enormously distended, their branches near the margins of the liver were visible on the surface, and they were filled with a fluid watery bile.

The mucous membrane of the stomach was rather spongy, of a reddish tint, and it contained half a pint of brown grumous matter, apparently secreted from its surface. We examined several portions of the mucous membrane both of the small and large intestines, but they presented nothing peculiar except a 
rather spongy texture, and on some parts a grey colour. The spleen was healthy but soft. The kidneys large and flaccid, tinged with bile throughout, particularly their lining membrane. The large vessels appeared healthy and the lumbar and other glands were not diseased. The bladder contained some yellow urine. The uterus was rather thick and round in its form, the cavity large, and the glands at the mouth of that organ put on the appearance of vesicles at first sight. They were distended with glairy almost gelatinous mucus of a yellow colour, which could with some force be squeezed from their orifices.

In this case it is possible that the disease of the pancreas was first set up, and had existed for some time, perhaps for some years, giving rise to the occasional rejection of food : latterly this disease had increased to the extent of pressing on the biliary ducts and retaining the bile both in the gall-bladder and in the liver. By this means morbid action, and perhaps deposit of some portions of the bile, took place in the substance of the liver, and it may be a question how far, in a constitution predisposed, this may lead to the formation of the peculiar modification of the tubercles observed in cases of this kind. As the disease proceeded to ulceration the bile made its escape partially into the intestines. 


\section{CASEIII.}

Pancreas diseased-malignant ulceration of the intestines - slight jaundice - fatty matter discharged from the intestines.

Jane Davis, aged 21, was admitted under my care into Guy's Hospital, July 13, 1831, labouring under anasarca, chiefly affecting the lower extremities. Her abdomen was also swollen, though there was no distinct fluctuation. Her countenance was sallow and her lips purple, and some of the whiter parts of the face slightly jaundiced. The conjunctiva was also very slightly yellow. She was by no means emaciated in her general appearance, but was restless, and preferred lying in a raised position. I learnt from strict enquiry that she had lived rather an irregular life, and had been much out of health for nearly two years, but for the last two months had shewn more decided illness. She was herself inclined to date her present complaints to having been exposed to wet on the 18th of last month, about the time when she expected her catamenia, after which she had shivering, followed by perspiration, and a constipated state of bowels, all which was attended by cough and some expectoration. In this state, but growing worse and worse, she had continued, till about five days ago, when her legs began to swell.

14. Her bowels had been eopiously opened without medicine. I saw the evacuations, they were abundant, of a pultaceous eonsistence, very deficient. in bile, and most dreadfully foetid. The chamber pot 
was completely filled, and on the surface was observed a thin scum like a thin film of grease which collected and coagulated as grease more decidedly upon the side of the ressel. The lower and more fluid parts of the contents of the ressel looked slightly tinged with blood. It was doubted whether some parts were not purulent. Placing the hand on the abdomen, the upper part was distended and rather tender, particularly towards the right side, where an indistinct hardness could be felt, somewhat resembling the liver.

Applicetur emplastrum ammoniaci $\overline{\mathbf{c}}$ hydrargyr. scobiculo cordis.

Habeat hydrag. $\bar{c}$ cret. gr. ij, pulver ipecac. comp. gr. ij, et infus. Gentian. $\bar{c}$ Soda ter die.

No marked change took place in the character of the stools or the urine, but the patient lost strength. The evacuations continued copious, fetid, and of a dark clay colour, with the fatty pellicle on the surface. The disease of the lips subsided but the tendency to jaundice increased, and on the 19th, after passing a restless night, she expired.

Sectio Cadaveris. - The whole body was decidedly tinged with bile, some yellow serum escaped from the abdomen when it was opened. The liver was immediately seen considerably enlarged from distension and of a dark olive green colour. The fundus of the gall-bladder projected, it was distended with bile of a dark green colour. The quantity which it contained 
could not have been less than four ounces, and the ducts were as large as the little finger till they entered the duodenum where the orifice was very small, and it required considerable force to make the bile pass into the intestines. The whole of the intestines were somewhat distended, and in several places, when viewed externally, their puckered and discoloured appearance plainly indicated that mischief had been going on within, and at one part of the small intestines, an actual perforation, large enough to admit the point of the little finger, had taken place, but a slight adhesion which gave way in the examination prevented the feculent matter from being effused.

The whole course of the intestines was now laid open, and fungoid excrescences and ulcerations were found distributed at irregular intervals from their commencement at the pylorus, to the termination of the colon. Whether any existed in the rectum, I am not quite certain. These ulcers might be traced throughout their whole progress. They began by small elevations, generally upon the edges of the convolutions of a light yellow or white substance, and those which had arrived at the size of a pea, generally had a depression in the centre as if from partial ulceration. The depressed part was softer than the surrounding edge, and if the tumour was squeezed, a whitish puriform or cerebriform matter issued from pores upon its surface. This, however, was better seen when the whole disc had increased to the size of a sixpence. About this time, or sometimes sooner, the surface lost its light and clean appearance, and 
became covered, sometimes with a sloughy mass, but more frequently with a dark grumous coat, apparently from blood, which had exuded, and become changed on its surface. The mass was now elevated nearly half an inch, the edges inverted or cup-shaped, and the centre either raised with the loose fungoid slough and blood, or if this had come away, was deeply excavated, going on in its progress to perforate the substance. In two instances, these fungoid ulcerations communicated immediately with large fungoid excrescences, probably glands situate externally to the intestine. One of these was close to the ileo-colic valve, where the external ulcer was black with grumous exudation, and formed the mouth of a cavity which would admit the finger into a mass involving the glands of the mesocolon. Another of the same kind, but less completely opening into the external diseased mass, occurred in a portion of the duodenum.

The mesenteric glands were involved in this disease, and the renal capsules, but more particularly the left, had suffered from the same affection.

The kidneys were healthy. The uterus was also healthy, but its appendages had suffered great irritation. One of the fimbriated extremities was completely bound down, and the orifice obliterated, and the ovaries were corrugated and contained vesicles in different states of disease.

In the liver no fungoid disease shewed itself, but its texture was natural, though gorged with bile. 
The pancreas was most deeply involved in the disease. It formed a hard mass near its head, and then a more healthy portion intervening, another hardened mass was seen near to the spleen, when another small portion remained healthy at its termination, so that it might be said to be occupied by two fungoid tubercles, which involved two thirds of its whole structure. The limits of these diseased masses were not distinctly defined, but they were of a more yellow colour than the rest of the organ, and destroyed the lobular structure of the gland.

The spleen was unusually small.

In the chest the same disease was found affecting the bronchial glands, and in the form of one round fungoid tubercle of the size of a moderate plum, in the apex of one of the lungs; this was imbedded completely in the substance, and was of a yellow white colour.

In this case, then, the disease of the pancreas, and the ulceration of the duodenum were to a very marked extent; but the obstruction of the common ducts was not so complete as in the two former instances; and it must also be observed, that the fatty excretion, though very well marked, was decidedly less abundant than in the other.

When we draw a comparison between the three foregoing cases, a very close analogy, or even identity, 
in many circumstances may be traced. In all of them chronic ailment terminated, sooner or later, in jaundice, and in all of them a great peculiarity in the character of the dejections existed. In the result of examination after death we have likewise some circumstances which exactly coincide in all-obstructed biliary ducts; the liver gorged with bile; fungoid disease attacking the head of the pancreas, and malignant ulceration on the surface of the duodenum. The question to be solved is, upon which of the diseased conditions indicated, or caused by these morbid changes, if upon either, the peculiarity of the alvine evacuations depended? That the obstruction of the biliary ducts, or even the total absence of all indication of biliary secretion is not usually attended by the same peculiarity in the evacuations, many cases which have been cautiously detailed by various authors, and many which we have all observed, bear sufficient testimony ; and I was therefore induced to ascribe it either to the existence of malignant disease, or to that disease being situated in the pancreas. That the simple fact of the malignant disease existing is not necessarily productive of such appearances in the feculent matter, I infer from cases both of that form of disease and of melanosis in the liver to a very great extent, being, within the scope of my own experience, unaccompanied by any such discharge, though the evacuations were submitted to the most rigid observation. That simple ulceration in the bowels, to any known extent, is not attended by any such symptom, I am led to believe from knowing that neither in the 
most extensive ulcerations of the large intestines in dysentery, nor in the worst cases of ulceration of the small intestines in fever, in diarrhœa, or in phthisis, does any thing of the kind usually occur. Whether, however, malignant ulceration of the mucous membrane is accompanied by this symptom, I cannot assert, though I have often seen most extensive malignant ulcers of the pylorus and of the rectum, where, though the evacuations were attentively observed, such fatty matter was not detected. As, however, a malignant ulceration of the membrane did exist in each of the foregoing cases, it is not impossible that this was the cause of this symptom; but we must bear in mind that such ulcerations are by no means uncommon, and that the phenomenon of which $I$ am speaking is uncommon, and that in each of the cases it, was accompanied by another morbid appearance, which is not common, namely, the malignant disease of the pancreas. The fact of the intestinal ulceration having, in each case, occupied the duodenum does, however, somewhat diminish the weight of this observation, for that is certainly not so frequent an occurrence. I own I was, after the first two cases had occurred, so much inclined to ascribe the fatty evacuation to the disease of the pancreas, that in the two following cases, in which I was consulted, in the autumn of 1830 , where disease of the pancreas was suspected, I was induced, from the absence of this symptom, to withhold my assent to the diagnosis, and I had no reason to regret my caution. In both instances $I$ had been requested by the physicians in attendance to see cases, 
of which the characters were very obscure, and in which pancreatic disease was suspected. Though of course with all that doubt which intelligent men, well acquainted with their profession, must feel when reasoning upon a disease of which, as yet, we possess such uncertain indications.

\section{CASE IV.}

The first case was that of a coachman, who had suffered the usual exposures of his situation, and had for several months been wasting and growing pallid, and complaining of constant deep-seated pain at the scrobiculus cordis going to the back. Although considerably emaciated at the time I saw him, no tumour was to be felt in any part of the abdomen. There was not the slightest appearance of jaundice, and no sickness at the stomach, nor was there any thing to indicate disease in the lungs.

It was under this absence of positive symptoms, that my friend had turned his views to the pancreas; and having been called upon to give my opinion, and not having an opportunity of seeing the evacuations at that time, I requested that they might be carefully observed, and if none of the fatty matter which I described should be discovered, I said that the data did not appear to me sufficiently distinct to found an opinion that the pancreas was diseased. I could not assert that it was not, but that probably some other change was slowly taking place in the abdomen, the 
nature of which was not plainly indicated. The stools were carefully watched, and none of the fatty matter appeared, he continued to lose flesh, and a hardness was perceptible at the pit of the stomach before be died.

On examination after death, tumours of a scirrhous character were found in the liver, in Glisson's capsule, and at the small curvature of the stomach, but the pancreas was perfectly healthy.

\section{CASE $V$.}

The second case was that of a man, aged 50, who had suffered deep-seated pain at the Scrobiculus cordis for several months, together with occasional palpitation of the heart, and abdominal pulsation. He wasted much and lost his colour, but no tumour, nor aneurismal enlargement could be felt. It was evident that the heart was diseased. No direct proof could be obtained of abdominal disease, and as nothing of the peculiar fatty appearance could be discovered in the alvine evacuations, I refused to assent to the opinion which had been entertained of the existence of pancreatic obstruction. About a month after I saw this man he gradually sunk, becoming slightly jaundiced a day or two before his death, having also had cough, with mucous expectoration.

Sectio Cadaveris. - Very general and old adhesions were found between the pleura costalis and the 
pleura pulmonalis, some parts of the lung were hepatized from old disease, while other parts were emphysematous, and some recent irritation was observable in the bronchial membrane. The heart adhered very closely to every part of the pericardium, and was enlarged in its substance universally. The mitral valves and the semilunar valves of the aorta were slightly diseased.

The liver contained a good deal of blood, which was distributed irregularly between the acini, so as to give a mottled or nutmeg appearance. The acini were light coloured, a little tinged with bile. The gall bladder was full of bile, but not distended beyond its natural size. The ducts were pervious; but it was with some difficulty we could make the bile pass from the gall-bladder to the duodenum, apparently owing to its tenacious condition. The pancreas was perfectly healthy, nor was there any material derangement in the other abdominal viscera.

Thus, then, these two cases seemed to bear testimony in favour of the diagnostic mark I had formed to myself; but it must be confessed that, though I was not wrong in my negative diagnosis in these cases, it is highly probable that the grounds on which I proceeded were fallacious, so that I should not now have introduced these cases, but as very strongly shewing the insufficiency of all our present diagnostic marks. I say that the grounds of my diagnosis were probably fallacious, for we shall find in the following 
cases almost all the circumstances of the disease, and of the dissection, including the fungoid disease of the pancreas, coinciding with the three I have previously Aetailed; but the peculiar symptom on which I had laid stress was apparently wanting; still, however, none of the cases will be foand to be exactly parallel.

I had no opportunity of seeing the first of these cases, though I was frequently consulted respecting it during its progress; but on most particular enquiries from Dr. Hull, of Montrose, who attentively watched it, I could not discover that the peculiar dejections had ever occurred. I must however say, that these enquiries were not made till after the patient's death, and that the observation of the medical men in attendance was never decidedly drawn to the question of fatty evacuations during life. I shall relate both the symptoms and the dissection as I received them at different times from Dr. Hull.

$$
\text { CASEVI. }
$$

no

Mrs. — aged 76, a lady of a full habit of body, has had a family of fourteen children, of whom the youngest is 34 years of age. She enjoyed excellent health till within six months, but has had occasional headaches, followed by bilious vomiting. She has taken a great deal of regular though not severe exercise, and has very rarely required any medicine whatever.

About the month of May, 1827, she began to feel 
occasional pains in her back and sides, coldness of her knees, slight giddiness, dimness of vision, specks floating before her eyes, occasional palpitation of the heart, particularly on first going to bed. Bowels sluggish, so as to require laxatives frequently, which always relieved her for some time.

These symptoms gradually increased, and, in the beginning of December, a constant uneasiness, with a sense of weight and distension of the stomach, with frequent eructations were added, and the palpitation became very distressing at night. A severe pain was experienced in the side, immediately under the right mamma, extending across the body, and down to the groin, sometimes on one side, sometimes on the other, often on both ; at other times, the pain was more severe in the back orer the kidneys. The pulse and tongue were quite natural; urine very scanty, of a pinkish colour, depositing a copious muddy deposit; the stools, except after medicine, always costive, but natural in appearance, though generally accompanied with a considerable quantity of mucus. The skin rather dry. No thirst. She complained much of a peculiar sensation, as if a button were placed under her stays, directly over the spinous process of the third dorsal vertebra, which spot was tender to touch, but presented no diseased appearance on examination. She was able to lie only on the left side, and experienced very acute pain and great difficulty in turning, or even altering 
her position in bed. She was oppressed with general lassitude, and indifferent to what were formerly her greatest amusements and chief occupations.

“ About the 26th of December, a considerable change took place in the appearance of the evacuations. The urine became of a much higher colour, and the stools bore a whitish clay-like aspect; her countenance assumed a bilious tinge. The pain of the side was much more acute, and fixed to a particular'spot. Nausea also was superadded to her other distressing feelings. The jaundice became gradually more and more confirmed, and towards the middle of April anasarca began to occupy the legs, and effusion took place in the abdomen.

" No material amendment occurred in any of the symptoms, but she grew gradually weaker, and died on the first of June, 1828.

"Sectio Cadaveris.-On opening the abdomen, we found the omentum unusually thickened, and extending down into the pelvis, to which it adhered : the omentum also formed the contents of an umbilical hernia, to which the patient had been long subject. On raising the omentum, the first object which presented itself was the gall-bladder enormously distended, and projecting above the stomach in the position in which the body lay. The gall-bladder exceeded three inches in diameter near its fundus, 
and in length measured six and a half inches, the cystic duct forming, with the gall-bladder, one straight pouch. The common duct was dilated to about one inch in diameter, and at its termination, where it should enter the duodenum, formed an absolute cul de $s a c$, resembling the point of the thumb of a glove, the whole completely tense from distention. When cut open, and the contents washed out, the continuation of the duct was found completely obliterated. The contents of the gall-bladder and duct together were about eight ounces of a thick perfectly black fluid, resembling paint.

“ The liver adhered slightly in two places to the diaphragm, but was in other respects quite healthy, and rather smaller than usual, but perfectly gorged with the same inspissated bile. The stomach empty, and healthy in appearance. The duodenum considerably thickened and somewhat contracted, the thickening gradually increasing from the pylorus to the part where the ductus choledochus usually enters, and where it came in contact with the pancreas, to which it adhered. The pancreas itself much larger than usual, and presenting a confused mass of scirrhous disease, involving the termination of the ductus choledochus in the same diseased structure, and becoming totally impervious.

“ The intestines, with the exception of the duodenum, healthy throughout ; the spleen, kidneys, ute- 
rus, and ovaries, were also healthy. As was to be expected, the intestines were loaded with fatty matter, deeply tinged with bile.

"The cavity of the abdomen contained about three quarts of thick fluid, resembling healthy bile, and from the noise in moving the body, there was no doubt a considerable quantity in the thorax also, but we were not permitted to examine."

Thus then we have a case which approaches so nearly to the first three, as to render it very difficult to explain why any essential symptom should differ; and indeed, the only point of distinction seems to be the condition of the duodenum, which, in all the other cases, had been affected by malignant ulceration, but in the present case was only united by the scirrhous disease to the pancreas. By this case, therefore, as far as the negative evidence of one fact can go, we are deprived of the assistance of the peculiar fatty dejection as diagnostic of interruption in the function, or lesion in the structure of the pancreas, unless associated with disease of the neighbouring intestine. At least, -if it has this origin when it occurs, it is not a constant symptom.

In the following case, malignant disease occupied a large portion of the pancreas towards its middle, but neither was the head of that organ diseased, nor 
was there any malignant ulceration in the intestine, though there was slight communication of the disease to its structure.

\section{w}

CASE VII.

Extensive Malignant Disease in the Mesentery and Glandular System, involving a large portion of the middle of the Pancreas.-Mucous Membrane of the Intestines not ulcerated.-No fatty dejection observed.

Mr. — aged 35, called upon me for advice, June 16th, 1831, being the subject at that time of abdominal tumours. There were several tumours, but the two chief masses were the one above and the other below the umbilicus. The upper mass which did not quite reach to the pit of the stomach, was about the size of the fist, and on its upper part there was an elastic portion, as if it were an intestine distended with air, lying upon the tumour. The lower mass lay below, and a little to the left of, the umbilicus, rising towards the margin of the ribs on the left side, but not to be traced as passing under them. This gentleman was able to walk about and transact his affairs as usual, and his complexion was good, but he complained of considerable loss of general strength, and this was increasing. He stated that ten months before, having previously enjoyed good health, he was supposed to labour under inflammation of the liver, for which he was bled, and other remedies were employed which gave him relief, but left 
him weak, and of this he never completely recovered; but he had not perceived the tumours till three months before the present time. I considered them to be connected with the omentum and peritoneum, but recommended that his bowels should be well opened, lest any part of the enlargement should depend on hardened faces, for he was himself impressed with a belief, by no means uncommon in such cases, that his bowels had not been fully acted upon.

1 heard no more of this patient till the 9th of April, 1832, when I was informed by Mr. Bassett of his death, and by his kindness I was afforded an opportunity of seeing the diseased parts. I learnt from that gentleman, that since I saw him he had been twice completely jaundiced; that his urine was high coloured, and his stools clay-coloured and yeast-like; but neither fatty matter nor mucus had been observed to be passed with them, and a few days before death they became very dark. He was greatly emaciated, and Mr. Bassett thought that the tumours had diminished lately, which he ascribed to the use of an ointment of iodine.

Sectio Cadaveris.-The liver was healthy in its general appearance, and was not gorged with bile; but there was on the convex surface, and near the margin of the right lobe, one scirrhous tuber about the size of a shilling, and on the same surface, further back, a few more, not larger than peas. They were as hard as cartilage, and did not appear to amalgamate in the slightest degree with the general sub- 
stance of the liver. About four more, of the size of marbles, were found deep-seated in the substance of the right lobe, and a considerable number of $a$. smaller size, some not larger than the smallest grain of mustard-seed.

The gall-bladder was greatly distended, at least six or eight inches in length, of a long oval shape, slightly tinged with green, but filled with colourless fluid.

It was found, that the upper tumour, which had been detected during life, consisted of a large moveable mass connected with the omentum, and hanging about two inches from the stomach, to the large curvature of which it was attached, at about one third from its pyloric extremity. The remaining portion of the omentum was almost completely free from the disease.

The lower tumour owed its form to an immense mass of a botryoidal figure, which arose from the root of the mesentery, and occupied the greater part of it. This mass rose up, and projected forwards in a very remarkable way, so as to displace the small intestines, most of which were driven towards the pelvis. This tumour was of great extent, involving the aorta and iliacs, and passing up along the course of the spine, and uniting with another large mass which occupied the situation of Glisson's capsule, involving the pancreas, and extending across to the kidneys, including the renal capsules. 
With regard to the pancreas itself, a portion of about three inches near to the spleen could be distinctly traced, though it was contracted by pressure to the size of the little finger, not apparently altered in structure, except by compression. As it passed towards the right side, it lost itself in the diseased mass, so that it was not possible to say where it ended or the disease began. I am, however, inclined to say, that the organ itself, or at least the cellular membrane uniting its lobules, was the seat of disease, because the duct, enlarged to the size of a small quill, was easily traced passing through the hard diseased mass, without having any of the natural substance of the pancreas around it. Another portion of the pancreas, at its right extremity, was also to be traced, of a tolerably natural texture.

The Renal capsules were both involved like the pancreas, so that it was difficult to say whether the disease actually occupied their structure or was superadded; but they appeared to me to be pervaded so completely by the disease, that in one of them a very small portion only retained its natural structure: of the other more remained perfect.

The spleen was healthy, as were the kidneys, except that a tubercle, like those in the liver, grew from the peritoneal covering of the right kidney.

On more careful examination of the biliary and pancreatic ducts, it was found, that the cystic duct was, like the gall-bladder itself, distended with a colourless 
fluid, and that the hepatic duct, which was also much enlarged, was empty, but stained with bile, while the common duct was small and contracted. This arose from the pressure of the diseased mass, which closed the orifice of the cystic duct, and produced a certain degree of stricture in the hepatic duct, but so as by no means to obstruct it completely.

The pancreatic duct was large during the greater part of its passage through the diseased mass, but was obstructed, so as not to allow the passage of a small probe when it reached the more healthy part of the organ : a probe could, however, be introduced from the orifice in the duodenum, and passed up the duct to the diseased portion.

The absorbent glands in different parts of the body, as in the axilla, groins, and neck, were enlarged, hard, and scirrhous. The disease certainly attacked the absorbent glands much more than is often the case in the true fungoid disease. The glands in the mesentery, near to the intestine, were scarcely if at all enlarged, but there was a line of hard white bodies along the mesocolon, between the great mass of disease and the intestines, which seemed to consist of hardened glands.

In every part, the diseased masses assumed a round or oval form, and an almost cartilaginous hardness; there was no cavity in any of them, but some of them were slightly creamy in their texture when cut into, and scraped with the scalpel, while in some several 
opake spots were observable, some of which had a cetaceous feel; these opake spots were distributed throughout the-tumours, and, in some instances, occupied their circumference or surface, so that when they were dried and cut through, they seemed to form an interrupted case. The tumours generally grew quite separate, so that, in this respect, being each of an oval form, they gave very greatly the idea of being enlarged absorbent glands.

The mucous membrane of the duodenum was decidedly diseased, though in an incipient state. It appeared as if the same malignant disease were establishing itself there beneath the membrane, and producing an irregular and slightly botryoidal surface, as if a few small particles of cauliflower were distributed upon it. The appearance ceased entirely at the pylorus. The head of the pancreas was not welded to the duodenum, nor was the structure of the intestine thickened generally : it was altogether free from any union with the diseased mass around which it turned. The mucous membrane of the other intestines, which I examined in various parts, was healthy.

Chest.-I had not an opportunity of examining the chest myself, but I understood that it was healthy, except that one small malignant tumour was found attached to the surface of the heart. 
After long and careful examination, it was found that the cystic and hepatic ducts had both been greatly dilated, while the common duct was small, the cause of which was the external tumour which almost closed the orifice of the cystic duct, and produced a certain degree of stricture upon the hepatic, but by no means so as to obstruct it completely.

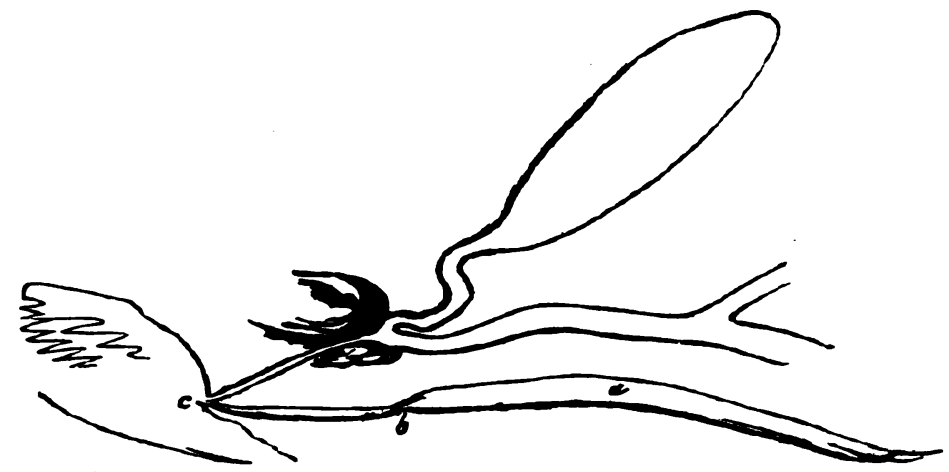

The pancreatic duct was large while it passed through the diseased mass ( $a$ ), but seemed to have been obstructed (b), (at least would not suffer the passage of a probe,) before it reached the more healthy part of the organ; but it admitted of a probe being introduced from the orifice in the duodenum (c).

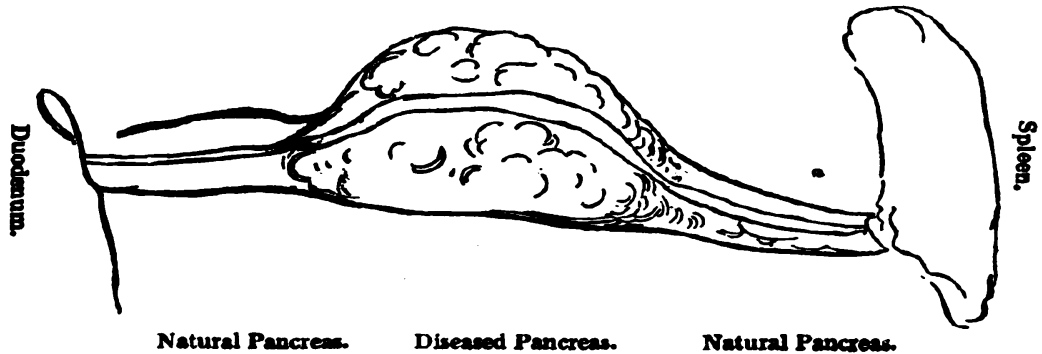


In this case, the disease was confined to the middle part of the pancreas, while that part towards the duodenum was tolerably, if not completely healthy, and the duodenum itself was not deeply involved in disease. It is, therefore, a question in this case, supposing it to be a fact that the fatty excretion did not exist, whether that might not depend upon the disease not occupying the head of the pancreas.

The two attacks of jaundice in this case, in all probability, arose from temporary causes, producing the complete closing of the hepatic duct, as there is no doubt it had been greatly distended at some former period, though pervious at the time of death.

The following case bears a strong analogy to those now under consideration, but the peculiar evacuations did not occur, or (which is very improbable) were not observed; and the only difference in the morbid appearance was, that the pancreas itself was not so decidedly the seat of the disease, but, at the same time, its duct was distended from obstruction, and the duodenum was ulcerated, so that this case will, more than any I have seen, militate against the unvarying occurrence of the symptom in connection with disease such as I have described. 


\section{CASE VIII.}

William Dempsey, aged 13, a sailor, who had been in various climates, both hot and cold, was admitted into Guy's Hospital, under my care, January 30th, 1828, being at that time the subject of wellmarked jaundice. It appeared that about thirteen weeks before, he first experienced a sense of weight, and a lassitude all over him, with occasional pain in the abdomen. About a week after, he perceived that his stools were white and his urine high coloured, his skin becoming at the same time yellow. These symptoms had all gradually increased-his bowels had been relaxed the whole time, and for the last eight or nine weeks he had experienced a sense of itching over the body and head. At the time of his admission, the colour of the jaundice was deep and dingy, the stools shewed that no bile passed by the intestines ; the urine was yellow, and did not coagulate. He was evidently much emaciated, though his appetite was unusually great. He complained of pain at the pit of the stomach, where an indistinct hardness was at that time to be perceived.

He was ordered to take a scruple of the extract of taraxacum three times a-day, and a few grains of blue pill, with half a grain of opium every night at bed-time. But after five days, the yellow colour of the skin appearing rather to increase, and the pain at the pit of 
the stomach to remain stationary, and the gums being slightly affected, he was cupped to the amount of twelve ounces from the pit of the stomach, and the blue pill was given up; the taraxacum alone being continued with the addition of a dram of the tincture of hop. After this, he appeared to improve a little; his bowels were less relaxed, and he complained of very little, except weakness and some uneasiness at the pit of the stomach.

It is not necessary, however, to detail all the circumstances which occurred during the progress of this case : it is sufficient for our present purpose to state, that the irregular and hard liver, as well as the distended gall-bladder, were easily recognized by the touch; that the colour of the skin remained dark and dingy; that he was frequently lost in a state of drowsiness; that emaciation went on; that a great tendency to the effusion of blood showed itself by different hemorrhages; that the stomach was often oppressed with flatus, and towards the end of life, vomiting became more frequent, particularly in the evening; that diarrhoea frequently occurred; and that the evacuations varied in colour, and were latterly very watery; but that on no occasion was the fatty matter detected, though the character of the stools was frequently noted.

He continued to express not only hope of ultimate recovery, but a strong conviction that he improved, 
to the last; and always, till the day before his death, insisted upon getting up.

About two o'clock in the morning of the 24th of August, he was seized with a most severe vomiting of dark-coloured fluid, which continued at intervals through the day, exhausting him greatly; but he remained sensible to the last, and in the evening, just after having drunk nearly half a pint of water, he died in perfect tranquillity.

Sectio Cadaveris, Aug. 25th, 1828.-His body was greatly emaciated, and the whole skin of a deep yellow colour. The integuments of the abdomen were so thin, that the tuberculated liver was distinctly felt as an irregular hard body, and on raising them, they were found quite deprived of all fatty matter. The cellular tissue was yellow, and the sternum and cartilages of the ribs looked yellow, owing to the colour of the surrounding cellular membrane. The serous membrane investing the lungs and the pleura costalis were yellow. An ounce or two of dark yellow serum was found in the cavity of the chest. The lungs were healthy, except some emphysematous dilatation of the air-cells, which, however, were not ruptured. The pericardium was yellow, and contained about two drams of a clear yellow fluid, quite mucilaginous in its consistence, drawing out in strings of an inch or two in length. The heart very small. 
The peritoneum was coloured with yellow, and the liver immediately shewed itself to be studded pretty regularly in every part with distinct yellow fungoid tubercles; the general substance of the organ being of a deep olive green. The fundus of the gallbladder of the size of a swan's egg was seen in its usual position, projecting beyond the margin of the liver.

On careful examination it was found, that no part of the liver was free from a distribution of the fungoid masses, varying from the size of a pea to that of a pigeon's egg or larger; and around the gallbladder a mass of yellow white fungoid matter, of the size of a lemon, occupied the acute edge of the liver, descending towards Glisson's capsule; this mass was becoming softened, and assuming a brownish yellow colour towards its centre. The tubera or diseased portions assumed a more or less rounded form, projecting on the surface of the liver, with depressions in their centres, and a number of opake spots were sprinkled over them; their edges both externally and on the cut surfaces appeared indeed at first view to be sufficiently defined; but on close examination, they were found blending imperceptibly with the structure of the liver itself, so that the fungoid bodies could not by any means be broken or dissected from the liver, being evidently rather of the nature of deposits, in the structure leading to degeneration of the substance of the liver, than mere adventitious or independent growths. 
The gall-bladder was opake, and full of an olive green dirty fluid, in which a certain quantity of grumous matter sank to the bottom, and contained a coagulum of blood about the size of a bean. The bile was far from bearing that excessively dark green colour, which I have sometimes found where the liver has been less diseased, and the icterus has depended more entirely on disease external to the liver.

It was impossible to trace distinctly either the hepatic, the cystic, or the common duct; for the mass of disease which was formed about Glisson's capsule by the liver, the head of the pancreas, and the absorbent glands completely involved these parts; but on opening carefully into the hepatic duct where it divides, we found it greatly enlarged, and filled, not with genuine bile, but with a watery, almost colourless, fluid, which on pressure was seen pouring abundantly from the smaller divisions of the bile ducts opening into the large duct.

The pancreas did not feel perfectly healthy, and its surface was sprinkled with opake specks, not unlike those on the surface of the hepatic tubercles. It was not enlarged, but at its head formed a mass with two or three absorbent glands, so that it was almost impossible to say which- was pancreas and which absorbent. This mass was altogether of rather a yellow colour, and where the duodenum passed over it, adhesion had taken place to that organ, and a process of softening and ulceration was set up upon the surface of 
the duodenum, communicating with a deep sinus in the mass, running towards the liver, in the direction of the biliary ducts. The duct of the pancreas was greatly enlarged, being not less than the largest swan's quill.

The stomach contained a large quantity of dark grumous fluid. The spleen was healthy. The kidneys perfectly so, except that on the surface of the tunic of the right kidney there was a small discoloured patch, which marked an incipient change arising from the contact of the diseased liver. In no other part was there the least tendency to the same alteration of structure.

On opening the head, the dura matter was seen of a bright yellow colour, and the arachnoid itself or the small quantity of serum beneath it was slightly tinged. The serum in the ventricles was tinged, and the choroid plexus looked slightly yellow. There was throughout the medullary portion of the brain a very manifest tendency to sanguineous congestion, and that peculiar mottled colour was evident, as well as the more defined vessels denoting, irregular and excessive distribution of blood.

It may be said that in all the cases of fatty evacuation from the bowels, which I have detailed, a state of general jaundice existed, and this is in fact true; but in the case of Davis, the jaundice was quite recent, and I once saw this symptom to a very consi- 
derable extent in a gentleman, who came to consult Dr. Babington, bringing with him, in a gallipot, the fatty matter, which he stated he had collected the night before, and which he said he passed almost every night, having, for a considerable time, laboured under a diarrhœe, which was always most troublesome soon after he first retired to bed. $\mathrm{He}$ was evidently much out of health, had lost flesh, and looked pallid, but had no appearance of jaundice. What was the result of his case, I never could ascertain; but it plainly shewed that the jaundice had no necessary connection with the peculiar excretion.

Taking then a general review of the foregoing cases, and recapitulating some of the foregoing observations, we find three instances only, in which the fatty evacuation existed; but in each of them so many morbid causes were combined, that it is necessary, by comparing them with other known facts, to reduce these causes as far as we are able, and in doing this we may observe-1st, that a great deficiency of the biliary secretion is well known very frequently not to produce the effect: 2dly, we also know that most extensive fungoid and melanotic destruction of the liver without jaundice is unattended by this symptom: 3dly, we know that extensive fungoid disease in the liver, with jaundice, does not produce it: 4thly, we know that the more simple and inflammatory diseases of the liver, which cause jaundice, are not characterized by these exacuations: 5 thly, we know that the extensive ulceration of the mucous membrane of the intestines, from diseases of 
other kinds, are not indicated by a discharge of fatty matter: and 6thly, we have every reason to believe that malignant ulceration frequently exists in various parts of the intestinal canal, from the pylorus to the rectum, but more particularly in these two parts, without this symptom. Thus then we bring the circumstances of the diseased structure, as far as they have hitherto attracted observation, in connection with this symptom, within a narrow. limit,-disease probably malignant of that part of the pancreas, which is near to the duodenum; and ulceration of the duodenum itself. These are the only two conditions, which can be traced as being peculiar to all the three cases, and there is good reason to believe that in one of the other cases which I have stated, where this symptom was wanting, the ulceration between the duodenum and the pancreas was also wanting; while in the next case a healthy portion of the pancreas intervened between the fungoid disease and the duodenum, and the mucous membrane of the intestine was not ulcerated ; and in the last case, the disease was probably rather seated in the absorbent glands than in the pancreas itself. In this last case, however, the duodenum was ulcerated, and the pancreatic duct greatly enlarged from obstruction, and therefore the case almost identical with those in which the fatty discharge had beenobserved.

I am well aware that deductions of this kind bear too much the appearances of sophistry to be very applicable to our reasonings on the phenomena of disease, and I freely own that they afford but 
slender conviction even to my own mind. I offer them, therefore, in no other view than as hints to be improved by future observers, and I will not even affect to decide, whence the peculiar fatty matter is derived : whether it is to be considered as a vitiated secretion from natural structures, which must here be chiefly mucous membranes; or as a discharge from the diseased and ulcerated parts; or as the product of defective digestion of alimentary matter, depending on the imperfect supply or irregular admixture of the biliary and pancreatic or other secretions, or on the perverted and impeded action of the duodenum.

The first case, which I have related, was so complicated in its symptoms from the existence of diabetes, that I was naturally led to doubt how far the general condition of the system induced by, or attendant on that disease, and, which is very frequently marked by fæcal evacuations of a peculiar character, might exert an influence in producing the fatty matter; but as I have seen a great number of diabetic cases, in which this symptom did not occur, and in no other case of diabetes observed it, and as diabetes was not detected, nor even suspected, in the other two cases of this evacuation which I have related, it seems probable that there is no essential connection between the two diseased actions, however much they may occasionally depend upon a common cause, or arise under the same constitutional predisposition. It may, indeed, at last, prove that the appearance to which I have been alluding, is but the 
result of ill-digested aliment; but even in that case, it will be very important to ascertain this fact, and to discover on what condition of the alimentary canal the different appearances, which are sometimes produced by fatty and oily substances, depend. The material, which most frequently, affords an opportunity for such observations to the physician, is castor oil, which seldom fails to produce peculiar appearances in the dejections, of which the most common is a number of yellowish white flakes disseminated through the liquid fæces; but at other times the oil passes, in part unchanged, particularly in children; sometimes a thin greasy pellicle is seen; and in some more rare cases, a great many small white spherical masses are formed in such a way, that they immediately suggest the idea of hydatids, and I have even seen them temporarily mistaken for such.

Should future experienceand observation servein any way to conmect the peculiar evacuation, which is at present under consideration, more decidedly with a diseased condition of the pancreas, or an imperfectaction of the duodenum, which would, in all probability, be associated with pancreatic derangement, it will possess much interest, as at this moment our knowledge of the symptoms attendant on the derangements of the pancreas, is very imperfect. Dr. Pemberton, in his straight-forward way of viewing disease, confesses this fact, reducing his diagnosis almost entirely to negative proofs, so that he says, " if we have deepseated pain in the region of the stomach, and more 
or less of sickness and emaciation, unattended by any other symptoms that mark an original disease of the stomach, of the posterior part of the liver, of the gallbladder or ducts, or of the small intestines, we may pretty securely pronounce that the disease is situated in the pancreas." Now, most undoubtedly, if we feel such confidence in our means of detecting all those diseases, which might produce the symptoms here mentioned, except disease of the pancreas, and ascertain that none of those diseases exist, we necessarily infer that the only remaining organ, is the seat of disease. This, however, requires such a range of knowledge, and such a confidence in a most extensive and difficult field of diagnosis, that it renders our conclusions most unsatisfactory, and unless we can arrive at something more tangible, our diagnosis will seldom rise beyond the standard of probable conjecture.

In the minor degrees of pancreatic derangement, perhaps the local pain is the only symptom from which, in the present state of our knowledge, we can expect to derive any information; but when the volume of the gland increases, or its structure undergoes considerable change, it is possible, that by its pressure it will produce some effects upon the functions of the surrounding organs, of which indigestion, sickness, and jaundice are the most prominent ; but as all these arise from a variety of causes, they are not calculated to throw much light on the disease; when combined, however, they naturally fix our 
attention upon one determinate point, in which the pancreas, from its situation, may be implicated. I am not aware of any peculiarity in the character of the sickness, or of the dyspepsia, or even of the pain which is yet proved to afford decided evidence of the pancreas being deranged, though it is highly probable that we shall hereafter discover peculiarities arising in these respects from the superabundant or deficient supply of the pancreatic secretion, which will be sufficiently marked. The profuse watery discharge in pyrosis, for instance, may well be conceived to have such an origin.

If the fatty dejections to which I have been referring, should indeed depend either on the derangement of the pancreas or of the duodenum, I should be far from expecting that such derangement had necessarily gone on to actual disorganization in all cases; for besides the individual to whom I have formerly referred, as bringing this peculiar secretion to Dr. Babington, I have been consulted respecting another train of symptoms, by a gentleman who had been in the East Indies, and who stated that, when in that country, and labouring under the effects of the climate, his stools had often borne the appearance of fat broth which had been suffered to grow cold, and in neither of these cases was there evidence of any advanced structural change.

With regard to the jaundice connected with pancreatic disease, the history of its gradual progress, 
and the dark dingy hue which it assumes, the emaciation with which it is attended, and the frequent opportunity which this affords of ascertaining the distended fundus of the gall-bladder long before death; these, if not marks decidedly diagnostic, are, at least, such as direct us to the probable existence of disease in the pancreas; but some more positive indication is still wanting, which may possibly be hereafter recognized in the peculiarities of the alvine evacuations.

There is but one further observation, which I would make in connexion with the cases which have been related. All of those in which the oily evacuation has been observed, have been cases of decided malignant, and (as far' as the pancreas is concerned) we might perhaps say, scirrhous, disease. Now it is a fact, which $I$ have observed in several cases, that the bile is very apt to undergo that change, which leads to the deposit of concretions of adipocire in the gall-bladder, in patients labouring under scirrhus, as females with scirrhous mammæ, for instance, where the disease either has or has not attacked internal organs, and I think it arises as a fair question, therefore, whether the peculiar appearances of the alimentary discharges may not depend on the same disposition, be it what it may, which leads to this unnatural deposit in the gall-bladder; and should this prove to be the case, the symptom would be diagnostic of the nature of the diseased action rather than of its seat. 